\title{
Implementación de la política nacional de acceso abierto en tres instituciones argentinas
}

Implementation of the national open access policy in three Argentine institutions

Agustin Zanotti

Universidad Nacional de Córdoba. Centro de

Investigaciones y Estudios sobre Cultura y Sociedad

(CIECS) - CONICET / Universidad Nacional de Villa

Maria. Instituto Académico Pedagógico de Ciencias Sociales,

Argentina

agustinzanotti@gmail.com

(iD https://orcid.org/0000-0002-7662-7593

Antonela Isoglio

Universidad Nacional de Córdoba. Centro de

Investigaciones y Estudios sobre Cultura y Sociedad

(CIECS) - CONICET / Universidad Nacional de

Córdoba. Facultad de Filosofía y Humanidades. Escuela de

Archivología, Argentina

antoisoglio@gmail.com

(iD https://orcid.org/0000-0002-0773-3499

Natalia Piccotto

Universidad Nacional de Córdoba. Centro de

Investigaciones y Estudios sobre Cultura y Sociedad

(CIECS) - CONICET / Universidad Nacional de

Córdoba. Facultad de Filosofía y Humanidades. Escuela de

Bibliotecología, Argentina

nataliapiccotto@hotmail.com

(iD https://orcid.org/0000-0002-3697-8508

\section{Resumen:}

En Argentina, el movimiento de Acceso Abierto (AA) comenzó a difundirse entre bibliotecarios e investigadores desde 2003. El gobierno nacional impulsó la vía verde al AA mediante la creación del Sistema Nacional de Repositorios Digitales y el mandato de colocar en disponibilidad pública los resultados y datos de investigación financiados con fondos estatales (Ley 26.899). A lo largo del artículo se examinó el proceso de implementación de esta política en tres instituciones del Sistema Nacional de Ciencia, Tecnología e Innovación: el Consejo Nacional de Investigaciones Científicas y Técnicas, la Universidad Nacional de Córdoba y la Universidad Nacional de Villa María. El estudio de caso se basa en una matriz comparativa con datos obtenidos de sitios web institucionales y de repositorios, entrevistas a informantes clave y análisis de textos legales. Ésta consta de cinco dimensiones: tecnología; procedimientos; contenidos; visibilidad y usabilidad; acciones complementarias. Los resultados demuestran que el crecimiento del AA expresa trayectorias particulares en cada institución, las cuales se manifiestan en formas organizativas y procesos de trabajo, volumen y tipo de contenidos depositados, pautas administrativas y tecnologías adoptadas, entre otros aspectos. Se observa aquí una interacción entre la política general, los contextos locales y los responsables de la implementación.

Palabras Clave: Acceso abierto, Políticas de información científica, Producción científica, Repositorios digitales, Argentina. 


\section{ABSTRACT:}

In Argentina, the Open Access Movement (OA) began to spread among librarians and researchers in 2003. The national government promoted the greenway to OA by creating the National System of Digital Repositories and the mandate to make available the results and research data financed with public funds (Law 26.899). Throughout the article we examine the process of implementing this policy in three institutions of the National System of Science, Technology and Innovation: the National Council for Scientific and Technical Research, the National University of Córdoba and the Villa María National University. The case study is based on a comparative matrix with data obtained from institutional and repository websites, interviews with key informants and analysis of legal texts. It consists of five dimensions: technology; procedures; contents; visibility and usability; complementary actions. The results show that the growth of OA expresses particular trajectories in each institution, which are manifested in organizational forms and work processes, volume and type of deposited content, administrative guidelines and adopted technologies, among other aspects. Here we observe an interaction between general policy, local contexts and those responsible for implementation.

KEYWORDS: Open access, Scientific information policies, Scientific production, Digital repositories, Argentina.

\section{INTRODUCCIÓN}

El artículo examina la implementación de la política nacional de acceso abierto (AA) en Argentina, tomando como casos de estudio a tres instituciones del Sistema Nacional de Ciencia, Tecnología e Innovación (SNCTI): el Consejo Nacional de Investigaciones Científicas y Técnicas (CONICET), la Universidad Nacional de Córdoba (UNC) y la Universidad Nacional de Villa María (UNVM).

El movimiento de AA comenzó a difundirse en el país entre bibliotecarios universitarios e investigadores hacia 2003, en confluencia con la creación del portal de publicaciones periódicas Biblioteca Electrónica de Ciencia y Tecnología (SECTIP, 2002). El Ministerio de Ciencia, Tecnología e Innovación de la Nación impulsó la vía verde al AA desde el año 2011, mediante la estrategia de conformación de una red interoperable de repositorios institucionales —el Sistema Nacional de Repositorios Digitales (SNRD) —, la creación de un Comité de Expertos, integrado por especialistas miembros de los repositorios que conforman el SNRD, y el establecimiento de políticas, estándares y protocolos comunes (MINCYT, 2011).

Otro avance significativo fue la sanción en 2013 de la Ley 26.899 Creación de Repositorios Digitales Institucionales de Acceso Abierto, Propios o Compartidos, la cual establece un mandato de AA de difusión pública de los datos y resultados de investigación, financiados con fondos estatales, en repositorios institucionales creados o modificados para tal fin. Comprende a todos los organismos del SNCTI. Los plazos estipulados son de seis meses desde su publicación para los resultados de investigación, y cinco años desde su recolección para los datos primarios (Argentina, 2013, art. 5). La ley fue reglamentada a finales de 2016, proporcionando definiciones adicionales y plazos máximos de entrada en vigencia.

Un estudio realizado en 2013 mostró que sólo 20 de un total de 57 universidades públicas nacionales tenían o estaban desarrollando un repositorio institucional y, en 2015, sólo siete integraban el SNRD (Fushimi, 2018). Los datos en abril de 2020 muestran una variación notable, con 40 instituciones adheridas al Sistema Nacional y 271.001 publicaciones (SNRD, 2020). De estos, ocho repositorios figuran entre los 1000 primeros dentro del Ranking Internacional de Transparencia de Repositorios (CSIC, 2019).

La creación del SNRD y otros instrumentos conexos, sumada al mandato de AA por la vía verde, constituyen una política nacional que se ha mantenido durante casi una década. Se acompaña de adecuaciones en cada universidad y organismo del SNCTI, relacionadas con la creación o modificación de repositorios digitales, la definición de pautas administrativas, la elaboración de políticas institucionales y la gestión de procesos de curación, depósito, preservación y difusión de los objetos digitales. El artículo informa acerca de las trayectorias de implementación de esta política nacional en los tres casos analizados. Para ello, utiliza una matriz comparativa con datos obtenidos a partir de sitios web institucionales y de repositorios, entrevistas a informantes clave y análisis de textos legales. La misma consta de cinco dimensiones: tecnología; procedimientos; contenidos; visibilidad y usabilidad; y acciones complementarias. La matriz refleja la política nacional de AA de Argentina, considerando las decisiones adoptadas por cada institución ejecutora. 


\section{REVISión DE LITERATURA Y ASPECTOS CONCEPTUALES}

Hace más de una década que universidades y organismos de ciencia, tecnología e innovación de la región debaten sobre los desafíos que la incorporación de las tecnologías digitales presenta en los procesos de producción, gestión y circulación del conocimiento científico. Uno de estos retos ha sido la implementación de modelos de publicación y difusión académica en AA.

Los antecedentes del movimiento de AA se remontan a las declaraciones emitidas por Budapest Open Access Initiative (2002, 2012), la Declaración de Bethesda sobre Publicación de Acceso Abierto (2003), la Declaración de Berlín sobre Acceso Abierto al Conocimiento en las Ciencias y las Humanidades (2003) y, en la región, la Declaración del Salvador sobre AA (2005). El AA a la literatura científica fue definido como

disponibilidad gratuita en la Internet pública, que permite a cualquier usuario leer, descargar, copiar, distribuir, imprimir, buscar o añadir un enlace al texto completo de esos artículos, rastrearlos para su indización, incorporarlos como datos en un software, o utilizarlos para cualquier otro propósito que sea legal, sin barreras financieras, legales o técnicas, aparte de las que son inseparables del acceso mismo a la Internet (Budapest Open Access Inicative, 2012).

Además, se encuentran iniciativas internacionales que han buscado precisar el significado de abierto cuando se aplica al conocimiento y las licencias que se adecuan a los términos del AA (Freedom Defined, 2007, Open Definition, 2015). Se distinguen principalmente dos rutas de AA: la vía dorada (gold $O A$ ), que implica la publicación de los resultados en revistas académicas de acceso abierto, y la vía verde (green $O A$ ), que abarca la difusión pública de los datos y resultados en repositorios digitales. Las publicaciones periódicas someten los trabajos a mecanismos de evaluación de pares (peer review), como el referato doble ciego o el referato público, para determinar su pertinencia y calidad. Los repositorios operan en general como fondos de archivo y colecciones digitales, sin que el depósito de contenidos constituya un sistema de validación de los conocimientos. ${ }^{1}$ Los repositorios pueden ser temáticos o institucionales, siendo este último caso el del presente estudio.

El movimiento de AA se sustenta en el supuesto de que la disponibilidad pública de las publicaciones científicas en internet posibilita su reutilización por parte de otros científicos (fomentando la colaboración y evitando la duplicación de esfuerzos), y de actores sociales externos a la academia, permitiendo que se beneficien de los hallazgos de las investigaciones de manera más amplia y directa (Abadal, 2012). Para los propios investigadores y las instituciones de pertenencia representa la posibilidad de obtener más visibilidad local e internacional, incrementar la citación de sus producciones y obtener reconocimiento, lo cual redunda en mayor acceso a financiamiento tanto estatal como privado (McDowell, 2018). Con respecto a las comunidades disciplinarias internacionales, el AA también ha sido asociado con la posibilidad de revertir la subrepresentación que tiene la producción científica de los países del Sur global, tal y como es el caso de la investigación latinoamericana (Aguado López y Vargas Arbeláez, 2016).

América Latina presenta una importante tradición de AA. Por un lado, existe una serie de iniciativas de colaboración y no comerciales, con apoyo gubernamental y un papel destacado de las universidades, en el mantenimiento de revistas gratuitas para lectores y autores. La Red de Bibliotecas Virtuales de Ciencias Sociales de América Latina y el Caribe de CLACSO, Scientific Electronic Library Online (SciELO), Latindex y la Red de Revistas Científicas de América Latina y el Caribe, España y Portugal (Redalyc), son algunos ejemplos destacados de redes que fortalecen a las revistas, a las que se sumó recientemente la iniciativa AmeliCA de Conocimiento Abierto para América Latina y el Sur Global (Becerril García et al., 2018). Las revistas latinoamericanas, en su mayor parte, no cobran cargos por procesamiento de artículos (APC), a diferencia del modelo de AA de las editoriales comerciales del Norte global (Fushimi y López, 2019).

Por otro lado, se registra una trayectoria de portales y repositorios digitales en AA, particularmente en las universidades latinoamericanas, y la existencia de nodos nacionales de recolección de las producciones científicas. En el ámbito regional, estos últimos están conectados en La Referencia, la Red Federada de 
Repositorios Institucionales de Publicaciones Científicas (Miguel, Bongiovani, Gómez y Bueno de la Fuente, 2013).

La perspectiva teórica del artículo parte del concepto de política pública, entendiendo que ésta implica una red de decisiones y el desarrollo de acciones a lo largo del tiempo, que configura campos en disputa, en lugar de un proceso lineal, coherente y necesariamente deliberado de diseño de un actor. Para ello, se distinguen tres momentos del ciclo de las políticas: formulación, implementación y evaluación, que en la práctica se encuentran vinculados en ciclos de retroalimentación (Dagnino, Thomas, Costa y Gomes, 2002). Este artículo está centrado en el análisis del momento de implementación de una política estatal de AA, atendiendo especialmente a las consideraciones de un enfoque de política bottom up (de abajo hacia arriba) en el cual

(...) decisões do dia a dia da implementação as que realmente requerem negociação e compromisso entre grupos poderosos e decisões-chave só podem ser tomadas quando existe uma percepção mais clara dos potenciais resultados da política à disposição dos "implementadores". (Dagnino et al., 2002, pp. 95-96).

El texto de la Ley 26.899 plasma este enfoque bottom up al establecer un mandato de AA que contiene lineamientos generales, como los plazos de depósito de los datos y los resultados de investigación en los repositorios digitales, pero no determina los procedimientos por los cuales las instituciones obtendrán, gestionarán, preservarán a largo plazo y otorgarán acceso abierto a dicha información digital. Por el contrario, la ley prevé que sean las entidades del SNCTI las que establezcan los mecanismos pertinentes a través de políticas institucionales de AA (Congreso de la Nación Argentina, 2013, art. 2). Precisamente, la reglamentación de la ley establece que la política de AA de cada institución deberá "indicar el recorrido" que hará su producción científico-tecnológica, financiada total o parcialmente con fondos estatales, a fin de quedar disponible públicamente a través del repositorio digital (MINCYT, 2016, art. 9.1).

\section{Metodología}

La presente indagación constituye un análisis descriptivo y comparativo realizado entre abril de 2019 y marzo de 2020. Las técnicas de recolección de datos utilizadas fueron:

- Entrevistas a informantes clave. Se realizaron tres entrevistas a responsables de gestión de cada repositorio digital, para indagar sobre su estado de avance, administración, proceso de trabajo e innovaciones recientes. Las entrevistas fueron administradas personalmente y a través de correo electrónico.

- Observación de portales web de repositorios institucionales. Prestaciones y funciones disponibles en cada uno de los casos seleccionados. Se extrajeron estadísticas del SNRD.

- Análisis de instrumentos de política institucional. Las resoluciones de creación de políticas institucionales son detalladas en cada caso, también la reglamentación vigente de la Ley 26.899 (MINCYT, 2016).

- Revisión de fuentes secundarias. Artículos científicos y ponencias publicadas, portales estadísticos y datos oficiales de las instituciones referidas. Permitieron la contextualización de los casos.

Con miras a reconstruir la diversidad de procesos de implementación existentes, las instituciones fueron seleccionadas de modo intencional en función de perfiles y diferencias estructurales.

El CONICET ha sido uno de los pioneros en la implementación de la Ley 26.899 y un referente para el resto de instituciones integrantes del SNCTI. Concentra más de 10.000 investigadores y 11.000 becarios, ambos con dedicación exclusiva a tareas de investigación. Posee 266 institutos descentralizados a lo largo del país, la mayoría de doble dependencia con universidades nacionales (CONICET, 2017a). 
La UNC es la mayor universidad del interior del país, la segunda en matrícula luego de la Universidad de Buenos Aires. Fundada en 1613, cuenta con 15 facultades, 136.000 estudiantes y 9.730 docentes, además de 145 centros e institutos de investigación, 25 bibliotecas y 17 museos (UNC, 2018).

La UNVM es una joven casa de estudios ubicada en el interior de la provincia de Córdoba, creada en 1995, con énfasis en la formación e investigación orientada al desarrollo local y regional. Cuenta con tres facultades, 10.000 estudiantes, más de 700 docentes y 6 centros de investigación (OIR, 2019).

Para analizar el proceso de implementación de la política nacional en cada caso, construimos una matriz multidimensional que recupera antecedentes referidos tanto a indicadores de evaluación de repositorios como a estudios de casos locales. Entre ellos, Serrano Vicente, Melero Melero y Abadal (2014) construyeron una síntesis de 32 indicadores que consta de cinco dimensiones. También consideramos los aportes de Fushimi (2018), quien realizó estudios de caso sobre tres universidades públicas argentinas. Asimismo, Ciolli y Disderi (2018) aportan una actualización de la discusión sobre indicadores que permiten ponderar la evolución de repositorios. La matriz de análisis quedó conformada con cinco dimensiones:

1. Tecnología: referida al tipo de plataforma digital seleccionada y sus principales prestaciones. Fueron consideradas tres variables: Software; Integración con sistemas informáticos universitarios; Estadísticas de uso obtenidas por el software de la plataforma digital utilizada.

2. Procedimientos: referida a la organización del proceso de trabajo y distribución de los flujos. Fueron consideradas cinco variables: Proceso de trabajo; Dependencia de gestión del repositorio; Políticas institucionales; Manuales de procedimientos; Autoarchivo de los objetos digitales en los repositorios por parte de los autores.

3. Contenidos: referida al volumen y composición de las colecciones que componen el repositorio institucional. Fueron consideradas tres variables: Cantidad de objetos depositados; Tipos de objetos depositados; Plan de Gestión de Datos.

4. Visibilidad y usabilidad: la primera se refiere a la posibilidad de que la plataforma digital sea encontrada en la web (Cordón García, Alonso Arévalo, Gómez Díaz y López Lucas, 2010), la segunda a la facilidad y efectividad de uso para los usuarios. Son aspectos que actúan de manera interrelacionada y se complementan. Para su análisis, fueron consideradas ocho variables: Licencia de uso de los objetos digitales; Enlaces desde el sitio web institucional; Sección de novedades y noticias; FAQs/ ayuda; Contacto; Adhesión al SNRD/ ROAR/DOAR; Optimización en motores de búsqueda; Utilización de identificadores persistentes para los objetos digitales.

5. Acciones complementarias: iniciativas conexas que se corresponden con una estrategia de AA. Fueron consideradas cinco variables: Sensibilización y capacitación a la comunidad; Existencia de otros portales AA; Comisiones asesoras; Normalización de la filiación institucional; Incentivo a publicar en AA en la evaluación.

\section{Resultados}

A continuación, se presenta el análisis de los resultados expuestos en la Tabla 1 y descritos en apartados que corresponden a cada uno de los casos de estudio. 
TABLA 1

Implementación de la política de acceso abierto en las instituciones

\begin{tabular}{|c|c|c|c|c|}
\hline \multicolumn{2}{|l|}{ Dimensiones } & \multirow{2}{*}{$\begin{array}{l}\text { CONICET } \\
\text { DSpace 6.0 Duraspace Open Source }\end{array}$} & \multirow{2}{*}{\begin{tabular}{|l} 
UNC \\
DSpace 5.5 \\
DuraSpace Open \\
Source \\
\end{tabular}} & \multirow{2}{*}{$\begin{array}{l}\text { UNVM } \\
\text { PhpMyBibli (PMB) PMB } \\
\text { Services Open Source }\end{array}$} \\
\hline \multirow{3}{*}{ Tecnología } & Software & & & \\
\hline & $\begin{array}{l}\text { Integración con } \\
\text { sistemas } \\
\text { universitarios }\end{array}$ & SIGEVA: unificación y transferencia de registros & $\begin{array}{l}\text { SIGEVA-UNC: } \\
\text { unificación y } \\
\text { transferencia de } \\
\text { registros }\end{array}$ & No \\
\hline & $\begin{array}{l}\text { Estadísticas de uso } \\
\text { obtenidas por el } \\
\text { software de la } \\
\text { plataforma digital } \\
\end{array}$ & Disponibles para administradores y usuarios & $\begin{array}{l}\text { Disponible para } \\
\text { administradores }\end{array}$ & $\begin{array}{l}\text { Disponible para } \\
\text { administradores }\end{array}$ \\
\hline \multirow[t]{5}{*}{2 Procedimientos } & Proceso de trabajo & $\begin{array}{l}\text { Coordinado en Red Federal de Especialistas en el } \\
\text { Repositorio Institucional }\end{array}$ & $\begin{array}{l}\text { Coordinado en } \\
\text { Nodos de la } \\
\text { Oficina de } \\
\text { Conocimiento } \\
\text { Abierto (OCA), } \\
\text { dependiente de la } \\
\text { Secretaría de } \\
\text { Gestión } \\
\text { Institucional } \\
\end{array}$ & $\begin{array}{l}\text { Centralizado en la } \\
\text { Biblioteca Central }\end{array}$ \\
\hline & $\begin{array}{l}\text { Dependencia de } \\
\text { gestión del } \\
\text { repositorio }\end{array}$ & $\begin{array}{l}\text { Oficina Técnica dependiente de la Gerencia de } \\
\text { Organización y Sistemas }\end{array}$ & OCA & $\begin{array}{l}\text { Biblioteca Central / } \\
\text { Área de informática }\end{array}$ \\
\hline & $\begin{array}{l}\text { Políticas } \\
\text { institucionales }\end{array}$ & Res. DIR 2705/2015 & $\begin{array}{l}\text { Res. HCS } \\
1365 / 2017\end{array}$ & En desarrollo \\
\hline & $\begin{array}{l}\text { Manuales de } \\
\text { procedimiento }\end{array}$ & $\begin{array}{l}\text { Sí, para especialistas y personal que realiza } \\
\text { pre-carga }\end{array}$ & no & NO \\
\hline & Autoarchivo & NO & NO & NO \\
\hline \multirow{3}{*}{3 Contenidos } & $\begin{array}{l}\text { Cantidad de objetos } \\
\text { Según SNRD (2020). }\end{array}$ & 89.595 AA: 44.789 & 5.813 AA: 5.813 & 1.039 AA: 1.039 \\
\hline & $\begin{array}{l}\text { Tipos de objetos } \\
\text { Según SNRD (2020). }\end{array}$ & 89.595 artículos & $\begin{array}{l}1.982 \text { tesis de } \\
\text { grado; } 1.450 \\
\text { documentos de } \\
\text { conferencia; } 796 \\
\text { tesis doctoral; } \\
775 \text { tesis de } \\
\text { maestría } 444 \\
\text { artículos; } 351 \\
\text { libros; } 8 \\
\text { conjuntos de } \\
\text { datos primarios; } \\
4 \text { partes de libro; } \\
2 \text { documentos de } \\
\text { trabajo; } 1 \text { otro } \\
\end{array}$ & $\begin{array}{l}986 \text { artículos; } 53 \\
\text { libros }\end{array}$ \\
\hline & $\begin{array}{l}\text { Plan de Gestión de } \\
\text { Datos }\end{array}$ & En desarrollo & NO & No \\
\hline \multirow{7}{*}{$\begin{array}{l}4 \text { Visibilidad y } \\
\text { usabilidad }\end{array}$} & $\begin{array}{l}\text { Enlaces desde el } \\
\text { sitio web } \\
\text { institucional } \\
\end{array}$ & Sí, un clic & Sí, un clic & Sí, dos clics \\
\hline & $\begin{array}{l}\text { Sección de } \\
\text { novedades y } \\
\text { noticias }\end{array}$ & Sí. & $\begin{array}{l}\text { NO, en el } \\
\text { repositorio. Sí en } \\
\text { el sitio web de la } \\
\text { ocA. }\end{array}$ & $\begin{array}{l}\text { No, en el repositorio. } \\
\text { Sí en el sitio web de la } \\
\text { Biblioteca Central. }\end{array}$ \\
\hline & FAQs/ ayuda & Sí & NO & No \\
\hline & Contacto & $\begin{array}{l}\text { Sí, Cuenta en Facebook, Twitter, Instagram, } \\
\text { YouTube, Soundcloud. }\end{array}$ & $\begin{array}{l}\text { Sí, formulario en } \\
\text { línea, correo } \\
\text { electrónico, } \\
\text { cuenta en Twitter } \\
\text { y Facebook. }\end{array}$ & $\begin{array}{l}\text { Sí, dirección, teléfono, } \\
\text { horario de atención. }\end{array}$ \\
\hline & $\begin{array}{l}\text { Adhesión al SNRD/ } \\
\text { ROAR/DOAR } \\
\end{array}$ & Sí & Sí & Sí \\
\hline & $\begin{array}{l}\text { Utilización de } \\
\text { identificadores } \\
\text { persistentes }\end{array}$ & Sí, Handle & Sí, Handle & No \\
\hline & $\begin{array}{l}\text { Licencias de uso de } \\
\text { los objetos } \\
\text { digitales }\end{array}$ & $\begin{array}{l}\text { Creative Commons } \\
\text { Atribución-NoComercial-CompartirIgual } 2.5 \\
\text { Argentina }\end{array}$ & $\begin{array}{l}\text { Creative } \\
\text { Commons }\end{array}$ & $\begin{array}{l}\text { Creative Commons } \\
\text { Atribución } 4.0 \\
\text { Internacional }\end{array}$ \\
\hline \multirow{5}{*}{$\begin{array}{l}5 \text { Acciones } \\
\text { complementarias }\end{array}$} & $\begin{array}{l}\text { Sensibilización y } \\
\text { capacitación a la } \\
\text { comunidad }\end{array}$ & Sí, capacitaciones presenciales y virtuales & $\begin{array}{l}\text { Sí, } \\
\text { principalmente } \\
\text { en los Nodos } \\
\text { ocA }\end{array}$ & $\begin{array}{l}\text { Sí, capacitaciones } \\
\text { regulares }\end{array}$ \\
\hline & $\begin{array}{l}\text { Otros portales de } \\
\text { contenidos en } \mathrm{AA}\end{array}$ & $\begin{array}{l}\text { Portal de Publicaciones Científicas y Técnicas del } \\
\text { Centro Argentino de Información Científica y } \\
\text { Tecnológica (CAICYT) }\end{array}$ & $\begin{array}{l}\text { Portal de Revistas } \\
\text { de la UNC, sitio } \\
\text { web de } \\
\text { comunicación } \\
\text { institucional de la } \\
\text { ocA }\end{array}$ & Dos revistas de AA \\
\hline & $\begin{array}{l}\text { Comisiones } \\
\text { asesoras }\end{array}$ & Sí & Sí & Sí \\
\hline & $\begin{array}{l}\text { Normalización de } \\
\text { la filiación } \\
\text { institucional }\end{array}$ & Res. DIR 515/2016 & $\begin{array}{l}\text { Res. HCS } \\
1125 / 2017\end{array}$ & No \\
\hline & $\begin{array}{l}\text { Incentivo a } \\
\text { publicar en AA en } \\
\text { la evaluación }\end{array}$ & En desarrollo & NO & NO \\
\hline
\end{tabular}

Fuente: elaboración propia. 


\subsection{Consejo Nacional de Investigaciones Científicas y Técnicas}

El Repositorio Institucional CONICET Digital se inauguró en agosto de 2015 a cargo de la Gerencia de Organización y Sistemas del organismo. Para su administración se creó una Oficina Técnica y un Comité Asesor, los cuales elaboraron conjuntamente las políticas institucionales, aprobadas por Resolución del Directorio $\mathrm{N}^{\circ} 2.705$ (CONICET, 2015).

Con respecto a la primera dimensión del análisis, CONICET Digital utiliza la plataforma DSpace, administrada por la comunidad de DuraSpace. A través del Módulo de Administración del Repositorio Institucional (MARI), se nutre de todas las producciones científico-tecnológicas depositadas por investigadores, becarios y personal de apoyo en el Sistema Integral de Gestión y Evaluación (SIGEVA), utilizado para la rendición de cuentas del organismo.

En cuanto a los procedimientos, el proceso de difusión de los trabajos científicos en CONICET Digital comprende varias etapas en forma manual y automática, que permiten mejorar la calidad descriptiva de cada objeto digital para la recuperación de la información (CONICET, 2019). El trabajo manual es realizado a través de una Red Federal de Especialistas de CONICET Digital, compuesta por alrededor de 50 miembros, quienes hacen curaduría digital de los trabajos producidos por el personal de su dependencia o colaboran con otras dependencias (Entrevista Oficina Técnica, 2019).

Con respecto a los contenidos, CONICET Digital publica versiones finales o post-prints que hayan atravesado un proceso de aprobación por una autoridad competente. En los casos en los cuales la difusión del artículo está limitada por derechos de autor o si el autor solicitó la exclusión del texto completo por motivos de confidencialidad, derechos de propiedad industrial o acuerdos previos con terceros, CONICET Digital publica sólo los datos bibliográficos (metadatos) de la producción académica. En el caso de los artículos con embargo editorial, el repositorio habilita automáticamente su disponibilidad al cumplirse el plazo de la restricción.

En 2016 se cargaron 10.000 artículos con más de diez curadores voluntarios (CONICET, 2017a). En el mismo año, se facilitó a los investigadores completar los registros en el SIGEVA a través del uso del DOI y se realizó la primera recolección de objetos digitales por parte del SNRD. Este repositorio se mantiene como el primer aportante en cantidad de registros al sistema nacional. En marzo de 2020 contaba con 89.595 objetos, todos ellos artículos, aunque el 50\% de ellos son de acceso restringido (44.570) o embargado (236) (SNRD, 2020). Cuando el acceso a los artículos se encuentra restringido por políticas editoriales, los usuarios pueden solicitar el texto completo con la autorización del titular de los derechos de explotación (Entrevista Oficina Técnica, 2019). Por último, el sitio Web institucional publica periódicamente un boletín acerca de CONICET Digital, el cual analiza, en términos de cantidad y calidad, la producción científica difundida a través de dicho medio.

En relación con la cuarta dimensión de visibilidad y usabilidad, la actualización de Dspace 6.0 en el 2016 permitió mejorar el proceso de migración de datos y el buscador de la plataforma. También se trabajó en la integración con Google Scholar y Altmetrics (CONICET, 2017b). La visibilidad y descarga aumentó drásticamente en función de estas mejoras: mientras en el último trimestre de 2017 registró 15.410 visitas, el último trimestre de 2019 alcanzó 1.310 .540 visualizaciones (Repositorio Institucional CONICET Digital, 2020). La licencia de uso que se otorga a los objetos digitales es Atribución-NoComercial-CompartirIgual 2.5 Argentina (CC BY-NC-SA 2.5 AR), con la excepción de los casos en los que sus titulares de derechos de autor indiquen explícitamente otra regulación (CONICET, 2015).

En cuanto a las acciones complementarias, los encuentros presenciales y virtuales de capacitación de los integrantes de la Red Federal de Especialistas de CONICET Digital buscan optimizar el trabajo, haciendo hincapié en la mejora de procesos y funcionalidades de la curación de metadatos, políticas y 
revisión de registros editoriales (Entrevista Red Federal de Especialistas RI, 2019). Asimismo, en 2016, el Directorio del CONICET aprobó una norma para establecer la forma correcta de declaración de filiación institucional del personal (CONICET, 2016) y los curadores digitales utilizan el Sistema Integral de Gestión de Organizaciones para la asociación entre los autores de los trabajos y sus respectivas instituciones de trabajo (Entrevista Oficina Técnica, 2019).

En 2017, comenzaron a construirse los lineamientos del Plan de Gestión de Datos (PGD), con talleres de capacitación presenciales y virtuales. Asimismo, se lanzó la plataforma PGD CONICET, integrada a los demás sistemas informáticos, para la generación de Planes de Gestión de Datos. Actualmente sólo se ha implementado una experiencia piloto en una convocatoria de subsidio a Proyectos de Investigación de Unidades Ejecutoras del CONICET. ${ }^{2}$

\subsection{Universidad Nacional de Córdoba}

El Repositorio Digital Universitario (RDU) de la UNC fue presentado el 29 de noviembre de 2011, como resultado de un proyecto de cooperación con la Universidad Complutense de Madrid (Nardi e Yrusta, 2012, Pacheco, 2019). Recibió financiamiento de la Agencia Española de Cooperación Internacional para el Desarrollo (AECID), organismo que también aportó a la creación del Portal de Revistas Científicas de la UNC y el repositorio digital de contenidos educativos Ansenuza, resultado de un convenio de cooperación con la Universidad de Santiago de Compostela.

Un paso para el crecimiento de estas políticas fue la creación de la Oficina de Conocimiento Abierto (OCA) en 2014 y el Área de Tecnología, Educación y Comunicación (ArTEC) dentro de la Secretaría de Asuntos Académicos (Pacheco, 2019). Mientras la primera tuvo originalmente por objetivo brindar asesoramiento y capacitación sobre AA, contribuir a mejorar el sistema de comunicación científica y dar visibilidad a la producción intelectual y los fondos antiguos localizados en sus archivos, bibliotecas y museos; la segunda incluyó tanto el programa de educación a distancia, como la producción de recursos educativos abiertos (UNC, 2014).

La disposición de creación de la OCA también determinó la composición de un Consejo Asesor integrado por los titulares de la Secretaría de Asuntos Académicos, la Secretaría de Ciencia y Tecnología y la Prosecretaría de Informática (UNC, 2014). Durante 2015, se trabajó en la conformación de nodos OCA en las unidades académicas y se realizaron ajustes tecnológicos para la incorporación del RDU al SNRD. La OCA ha funcionado administrativamente bajo la órbita de la Secretaria General desde 2016 y de la Secretaría de Gestión Institucional desde 2019 (UNC, 2016, 2019c).

Con respecto a la primera dimensión del análisis, referida a la tecnología, el RDU utiliza la plataforma Dspace desde su creación. Al momento de realizar esta investigación comporta la versión 5.5. Aunque esta plataforma brinda estadísticas de uso del repositorio, informantes clave expresaron que éstas presentan alto margen de error, por lo que están siendo revisadas por el personal de informática (UNC, 2019a).

A fines del 2019, la OCA presentó la herramienta informática Migración de Metadatos y Archivos Digitales (MMAD), desarrollada conjuntamente con la Prosecretaría de Informática de la universidad, para la transferencia de las publicaciones depositadas por los docentes-investigadores en el SIGEVA de la UNC al RDU (UNC, 2019b).

En segundo lugar, la administración del repositorio digital está centralizada en la OCA, pero la gestión de los contenidos se basa en un sistema coordinado mediante nodos en las diferentes unidades académicas de la universidad, los cuales se localizan principalmente en las bibliotecas. Actualmente son alrededor de 37 las personas que trabajan en la gestión del RDU, sumando el personal de la OCA y los nodos respectivos en las unidades académicas (Entrevista OCA, 2019). Estos últimos no cuentan con un manual de procedimientos para desarrollar sus actividades, sino que siguen los lineamientos generales contenidos en el documento 
de política institucional. En 2017, el Consejo Superior de la UNC aprobó las Políticas Institucionales de Acceso Abierto para Publicaciones de la Universidad Nacional de Córdoba (UNC, 2017b). Establece las especificidades respecto de la política de contenidos, depósito, derecho de autor, estadísticas, resguardo de datos personales, preservación digital y eliminación de registros.

En tercer lugar, según las políticas institucionales, pueden depositarse en el RDU tanto los resultados de la producción científica y tecnológica — artículos de investigación, ponencias, libros, capítulos de libros, metadatos de patentes - como otros trabajos que forman parte de la actividad académica y cultural informes técnicos, tesis de maestría y doctorado, trabajos finales de grado y especialización, trabajos de prácticas profesionales supervisadas, pósters, películas, audiovisuales - o que constituyen datos en los que se basan dichas actividades - fotografías, planos, mapas, diapositivas, imágenes satelitales, radiografías, entre otros. Sin embargo, la universidad aún no ha establecido lineamientos para el tratamiento y la difusión de los datos primarios, ya que las políticas institucionales refieren solo a publicaciones. Con respecto a la cantidad de objetos digitales disponibles en el SNRD, el RDU ocupa la novena posición, con un total de 5813 (SNRD, 2020).

En cuarto lugar, la visibilidad directa se constata a través de distintos mecanismos. Desde el sitio web institucional de la UNC se puede acceder al repositorio digital con solo un clic sobre el ícono correspondiente. El RDU brinda diversas vías de contacto con la OCA: formulario en línea, dirección de correo electrónico de la OCA, cuenta institucional en Twitter y Facebook. Sin embargo, el repositorio no brinda al usuario un espacio de preguntas frecuentes (FAQS) o ayuda para orientar la recuperación de la información, más allá de los filtros de búsqueda disponibles. Tampoco se brindan noticias o novedades en esta plataforma digital. Éstas son publicadas en el sitio web de comunicación institucional de la OCA. Sin embargo, el repositorio no presenta un enlace al sitio web de la OCA. Además, la comunicación visual en ambas plataformas digitales no es homogénea, ya que se evidencia un uso dispar de isologotipos, colores, tipografías. Igualmente, algunos nodos en las dependencias universitarias elaboran carátulas institucionales para los archivos digitales que difundirán en el RDU, sin contar con criterios comunes; mientras que otros los depositan sin edición previa. El RDU es el único repositorio digital de la universidad adherido al SNRD. Los contenidos almacenados en el RDU son recolectados por este y, a su vez, forman parte de La Referencia. La política institucional establece que todo objeto digital a depositar en el RDU debe contar con una licencia de uso similar a Creative Commons, pero no indica qué combinación de la serie opera por defecto (UNC, 2017b).

En quinto lugar, se observan acciones complementarias a la implementación del repositorio institucional, como la disposición acerca de la obligatoriedad de mencionar la pertenencia a la UNC en todas las producciones académicas realizadas por empleados o personas que tienen como lugar de trabajo en esta universidad, así como la forma en la cual esta filiación debe ser declarada (UNC, 2017a). Destaca asimismo su Portal de Revistas, con cerca de 80 publicaciones periódicas en AA en la plataforma Open Journal System (OJS), así como iniciativas de Recursos Educativos Abiertos (REA) y actividades de promoción a cargo de facultades.

\subsection{Universidad Nacional de Villa María}

En el caso de la UNVM, la tarea de puesta en marcha del Repositorio Digital (RD) se inició en 2014 con la instauración del Programa Producción y Circulación del Conocimiento en la Universidad Pública (PyCCUP), creado en conjunto por los tres Institutos que componen la universidad: ciencias básicas y aplicadas, ciencias sociales y ciencias humanas (IAPCByA, 2014, IAPCS, 2014, IAPCH, 2014). Se creó una comisión de asesoramiento compuesta por docentes, investigadores, técnicos y personal de gestión, y se avanzó en tareas de diagnóstico, capacitación, conciliación de intereses y adecuaciones institucionales (Grasso, Pagola y Zanotti, 2019). Junto con ello se formuló una primera versión de las políticas del repositorio institucional, las cuales se encuentran aún en desarrollo. Algunas ya están implementando de 
hecho, como la utilización de licencias Creative Commons Atribución 4.0 Internacional (CC BY 4.0).

En cuanto a la tecnología, a diferencia de los casos precedentes, se optó por la plataforma PhpMyBibli (PMB), que ya venía siendo empleada como Sistema Integrado de Gestión Bibliotecaria (SIGB). La elección de PMB permite integrar y simplificar las tareas al trabajar dentro de un único sistema (Gómez Rodríguez, 2016). Se argumenta que los sistemas actuales permiten cubrir todos los requerimientos del repositorio digital, el cual puede visualizarse en un subsitio separado o dentro del conjunto de las colecciones disponibles. Con respecto a la integración con otros sistemas universitarios, actualmente no está disponible.

El proceso de trabajo se organiza a partir de una persona dedicada dentro del equipo de la Biblioteca Central. Se cuenta asimismo con el soporte técnico del Área de Informática, dependiente de la Secretaría de Planificación Técnica, Servicios y Mantenimiento. Al momento del relevamiento, se contaba con 1.039 objetos digitales en acceso abierto (SNRD, 2020).

Estos son procesados a solicitud de los institutos, en caso de eventos, tesis o trabajos finales de grado. Asimismo, se realizan búsquedas periódicas de objetos producidos por investigadores y docentes de la institución, los cuales son enlazados o duplicados en el repositorio. La curación de los objetos incluye la creación de una portada institucional y, en algunos casos, tareas de edición y formato. Debido a la centralización de las actividades, no se han elaborado hasta el momento manuales de procedimientos, aunque se considera que la documentación puede contribuir en la calidad de los procesos. Los objetos son cosechados por el SNRD y están accesibles por esta vía desde marzo de 2015. El RD también está registrado en los principales directorios internacionales.

En cuanto a las tareas de visibilidad del repositorio, se mantiene una sección de novedades e información de contacto dentro del sitio web de la Biblioteca Central. No se cuenta a la fecha con una sección de preguntas frecuentes ni ayuda o instrucciones para los usuarios. Otras mejoras de visibilidad y posicionamiento, tanto tecnológicas (optimización en motores de búsqueda), como institucionales (acceso desde el dominio principal o normalización de la filiación institucional), no han sido todavía abordadas. Tampoco se implementaron identificadores persistentes al momento del relevamiento.

La gestión del RD incluye otras acciones complementarias como el acompañamiento en la semana internacional del AA y capacitaciones periódicas. El Instituto Académico Pedagógico de Ciencias Sociales creó asimismo dos revistas académicas bajo la plataforma (OJS): Raigal. Revista interdisciplinaria de ciencias sociales, con revisión por pares, y Sociales investiga. Escritos académicos de extensión y docencia, para la publicación de comunicaciones y resultados de investigación.

\section{Discusión}

La descripción de los tres casos permite una comparación de los datos obtenidos sobre una matriz de cinco dimensiones. Este análisis posibilita avanzar hacia una mejor comprensión de la diversidad de trayectorias seguidas por las instituciones del SNCTI en el marco de una política de AA por la vía verde.

En cuanto a la tecnología, la mayor parte de los repositorios en la actualidad utilizan plataformas digitales de código abierto, cuyos soportes y mejoras se nutren de comunidades locales y globales. Esto permite su auditabilidad y adaptación para nuevos usos. De acuerdo con las directrices del SNRD, los repositorios de AA deben seguir estándares de interoperabilidad, como el protocolo Open Archive Initiative-Protocol for Metadata Harvesting (OAI-PMH), que permite la transmisión de metadatos codificados en Dublin Core (SNRD, 2019). La interconexión entre los repositorios y los sistemas informáticos universitarios permite automatizar algunas etapas del proceso de carga de objetos digitales, a partir del depósito realizado por docentes e investigadores en otras plataformas. Esto agiliza la gestión de grandes volúmenes de información y reduce el tiempo de puesta en disponibilidad pública. 
En lo referido a procedimientos, se hallaron tres configuraciones posibles de los procesos de trabajo: descentralizada, coordinada o centralizada. La primera es el caso de universidades de mayor tamaño, en donde los repositorios de las unidades académicas generan un volumen considerable y son por ello administrados de manera independiente. Es el caso de la Universidad de Buenos Aires, no incluida en el estudio. La segunda implica la descentralización del proceso de carga y decisiones relacionadas, mientras que los contenidos son depositados en un repositorio administrado por una dependencia central. Ello implica el mantenimiento de una red, así como el establecimiento de procedimientos estandarizados. Es el caso del CONICET y la UNC. El tercer caso es el de universidades de menor tamaño, en donde sus servicios de información son concentrados por una biblioteca central. Es el caso de la UNVM. Junto con cada configuración, encontramos diferentes dotaciones de personal y asignación de recursos. En los tres casos los contenidos son curados por personal especializado de la institución y el autoarchivo por parte de los autores no está disponible.

En cuanto a los contenidos, sus indicadores determinan el ciclo de vida del repositorio, esto es, su nivel de actividad a lo largo del tiempo (Fushimi, 2018). Permiten inferir usos, ya que los repositorios pueden contener una diversidad de formatos textuales, de datos y multimedia: artículos de investigación en versión final, pre y post-print, libros completos o capítulos de libros, ponencias y disertaciones, literatura gris como tesis y trabajos finales de grado, bases de datos e imágenes, entre otros (Suber, 2015). Se observa que mientras el CONICET se centra principalmente en los artículos revisados por pares, las universidades hacen un uso más diverso que incluye otros formatos, como ponencias aceptadas en conferencias, trabajos finales de grado y tesis de posgrado. Además, el CONICET comenzó la implementación de planes de gestión de datos a partir de una experiencia piloto, lo que no pudo hallarse en las universidades analizadas. Cabe mencionar aquí la importancia de que todas las instituciones concreten los requerimientos que establece la Ley 26.899 para anticipar el tratamiento que recibirán los datos a ser difundidos en AA en los repositorios institucionales.

Los casos analizados muestran que la visibilidad y usabilidad de los repositorios son aspectos que todavía no ocupan un papel central en su gestión. Aún más lejos se encuentran las prácticas de comunicación pública de la ciencia de los contenidos que se abren. En este sentido, destacamos algunas iniciativas conexas que completan la política nacional de AA y potencian sus aplicaciones, entendiéndose no como un fin en sí mismo, sino un medio para una mayor reutilización e impacto social del conocimiento científico. Éstas incluyen: tareas periódicas de sensibilización y capacitación a la comunidad; la creación de otros portales de revistas, datos o REA, el mantenimiento de comisiones asesoras de seguimiento y la implementación de incentivos a publicar en AA, entre otras.

\section{Conclusiones}

El modelo de publicación y difusión en AA se extiende en la región, representando formas alternativas de circulación del conocimiento científico. En Argentina, las acciones de promoción de la vía verde, llevadas a cabo por el Estado desde 2011, tuvieron el efecto de consolidar un proceso incipiente de creación de repositorios digitales institucionales. Luego de casi una década, se observan trayectorias particulares de implementación en las instituciones y los organismos del SNCTI. El análisis sobre tres casos permite observar una diversidad en formas organizativas y procesos de trabajo, volumen y tipos de contenidos depositados, políticas institucionales y tecnologías adoptadas, entre otros aspectos.

Desde un abordaje de análisis de políticas, estas trayectorias son el resultado de un proceso interactivo en el cual la política estatal se relaciona con los contextos locales y los responsables de su implementación. En este sentido, la puesta en marcha inicial de los repositorios ha dado paso a un proceso continuo de curaduría y gestión de contenidos, que requiere definiciones y procedimientos por parte de los actores involucrados. Determinados aspectos se encuentran aún en una etapa incipiente de implementación, como es el caso de los datos primarios de investigación. 
Una cuestión a profundizar es que, mientras la Ley 26.899 sentó definiciones para el AA por la vía verde, no hubo en el periodo una iniciativa similar que se pronuncie sobre la vía dorada. Los casos analizados muestran, sin embargo, avances en esta dirección sin una instancia de coordinación central, y sostenidas económicamente con recursos propios de las instituciones.

Son muchos los retos de las instituciones del SNCTI para potenciar las estrategias de AA, con el objeto de extender el impacto social de los conocimientos producidos. Si se busca aprovechar las posibilidades que brindan las tecnologías digitales para generar espacios abiertos de producción y uso de conocimientos, la inclusión del AA dentro de políticas más amplias de ciencia abierta, que implican la colaboración entre científicos profesionales y legos, es uno de los desafíos para los próximos años.

\section{Agradecimientos}

Agradecemos especialmente a los equipos de la Oficina Técnica de la Gerencia de Organización y Sistemas del CONICET, la Oficina de Conocimiento Abierto de la UNC y la Biblioteca Central de la UNVM.

\section{REFERENCIAS}

Abadal, E. (2012). Acceso abierto a la ciencia. Barcelona: Editorial UOC. Recuperado de: https://core.ac.uk/downlo ad/pdf/11889005.pdf

Actis, G. y Carlino, L. (2018). Plan de Gestión de Datos en CONICET: análisis, experiencia y desafíos. VII Conferencia Internacional BIREDIAL-ISTEC'17, 2, 3 y 4 de octubre de 2017, La Plata, Argentina. En VII Conferencia Internacional BIREDIAL-ISTEC'17. Memoria final. La Plata: Universidad Nacional de La Plata. Recuperado de http://sedici.unlp.edu.ar/handle/10915/67505

Aguado López, E. A. y Vargas Arbeláez, E. J. (2016). Reapropiación del conocimiento y descolonización: el acceso abierto como proceso de acción política del sur. Revista colombiana de sociología, 39(2), 69-88. Recuperado de h ttp://dx.doi.org/10.15446/rcs.v39n2.58966

Becerril García, A., Aguado López, E., Batthyány, K., Melero, R., Beigel, F., Vélez Cuartas, G., y Torres, J. (2018). AmeliCA: Una estructura sostenible e impulsada por la comunidad para el Conocimiento Abierto en América Latina y el Sur Global. México: Redalyc, Universidad Autónoma del Estado de México, CLACSO, Universidad Nacional de La Plata, Universidad de Antioquia.

Berlin Declaration on Open Access to Knowledge in the Sciences and Humanities. (2003). Max-Planck-Gesellschaft. Recuperado de https://openaccess.mpg.de/Berlin-Declaration

Budapest Open Access Initiative. BOAI. (2002). Iniciativa de Budapest para el Acceso Abierto. Recuperado de https:/ /www.budapestopenaccessinitiative.org/translations/spanish-translation

Budapest Open Access Initiative. BOAI. (2012). Diez años desde la Budapest Open Access Initiative: hacia lo abiertopor defecto. Recuperado de https://www.budapestopenaccessinitiative.org/boai-10-translations/spanish

Ciolli, M. E. y Disderi, J. (2018). Aplicación de criterios para la evaluación de un repositorio institucional en implementación. El caso del Repositorio Digital Universitario (RDU) del CRUC-IUA. Córdoba: Universidad de la Defensa Nacional. Recuperado de https://rdu.iua.edu.ar/handle/123456789/1825

Cordón García, J. A., Alonso Arévalo, J., Gómez Díaz, R. y López Lucas, J. (2010). Las nuevas fuentes de información: información y búsqueda documental en el contexto de la web 2.0. Madrid: Pirámide.

Dagnino, R.; Thomas, H. E.; Costa, G. y Gomes, E. J. (2002). Metodologia de análise de políticas públicas. En G. Costa y R. Dagnino (Org.). Gestão estratégica em políticas públicas (pp. 51-113). Campinas: Universidade Estadual de Campinas.

Declaración de Bethesda sobre Publicación de Acceso Abierto. (2003). ICTlogy. Recuperado de http://ictlogy.net/a rticles/bethesda_es.html 
International Seminar on Open Access. (2005) Declaración de Salvador sobre Acceso Abierto: la perspectiva del mundo en desarrollo. International Seminar on Open Access for Developing Countries, 21 y 22 de septiembre de 2005, Salvador, Brasil. En D. Babini y J. Fraga (comps.). Edición electrónica, bibliotecas virtuales y portales para las ciencias sociales en América Latina y El Caribe (pp. 237-238). Buenos Aires: Consejo Latinoamericano de Ciencias Sociales. Recuperado de http://biblioteca.clacso.edu.ar/clacso/biblioteca/20110818115141/Decla_S alvador.pdf

Freedom Defined. (2007). Definition of free cultural works. Recuperado de https://freedomdefined.org/Definition

Fushimi, M. S. (2018). Desarrollo de repositorios digitales institucionales en las universidades nacionales en Argentina, periodo 2004-2015. Tesis de Maestría en Ciencia, Tecnología y Sociedad. Bernal: Universidad Nacional de Quilmes. Recuperado de http://ridaa.unq.edu.ar/handle/20.500.11807/887

Fushimi, M. S. y López, F. A. (2019). Las vías abiertas de América Latina. Palabra clave, 9(1), e076. Recuperado de h ttps://www.palabraclave.fahce.unlp.edu.ar/article/view/PCe076

Gómez Rodríguez, G. (2016). SiGB usado como repositorio: El caso de la UNVM-PMB. En 2 Workshop Nacional de Tecnologías e Infraestructuras de la Información para Bibliotecas y Centros de Documentación e Información, Lanús, Argentina.

Grasso, M., Pagola, L., Zanotti, A. (2019). Implementación de una estrategia de Acceso Abierto en la Universidad. El caso de la UNVM, Argentina. Biblios. Revista de bibliotecología y ciencias de la información, 74(1), 79-89.

International Doi Foundation. (2016). Handbook. Recuperado de https://www.doi.org/doi_handbook/TOC.html

McDowell, Z. J. (2018). Disrupting academic publishing: questions of access in a digital environment. Media practice and education, 19(1), 52-67.

Miguel, S. E.; Bongiovani, P.; Gómez, N. y Bueno de la Fuente, G. (2013). Situación y perspectivas del desarrollo del Acceso Abierto en Argentina. Palabra clave, 2(2), 1-10. Recuperado de https://www.palabraclave.fahce.unlp.e du.ar/article/view/PCv2n2a01

Nardi, A. M. e Yrusta, L. (2012). Oficina de conocimiento abierto: un modelo para institucionalizar el acceso abierto en las universidades. El profesional de la información, 21(6), 633-637. Recuperado de http://hdl.handle.net/11 $086 / 1367$

OIR - Observatorio Integral de la Región. (2019). UNVM en Cifras. Recuperado de http://oir.unvm.edu.ar/estadist icas-academicas/unvm-en-cifras/\#tab-id-3

Open Definition. (2015). Open Definition 2.1. Recuperado de http://opendefinition.org/od/2.1/en/

Pacheco, M. (2019). Políticas de acceso abierto en Córdoba, proceso de institucionalización: La producción del texto de la política. En Actas de las V Jornadas de Intercambio y Reflexión acerca de la Investigación en Bibliotecología. La Plata: Universidad Nacional de La Plata. Recuperado de http://www.memoria.fahce.unlp.edu.ar/library?a= $\mathrm{d} \& \mathrm{c}=$ eventos\&d$=\mathrm{Jev} 11025$

Serrano Vicente, R.; Melero Melero, R. y Abadal, E. (2014). Indicadores para la evaluación de repositorios institucionales de acceso abierto. Anales de documentación, 17(2), 1-12. Recuperado de http://dx.doi.org/10.6 018/analesdoc.17.2.190821

Suber, P. (2015). Acceso abierto. Toluca: Universidad Autónoma del Estado de México.

UNC. Universidad Nacional de Córdoba. (2018). Sintesis estadistica 2018. Recuperado de https://www.unc.edu.ar/ sites/default/files/unc_sintesis_estadistica_2018.pdf

\section{FuENTES}

Argentina. (2013). Ley $N^{\circ}$ 26.899. Recuperado de http://servicios.infoleg.gob.ar/infolegInternet/verNorma.do?id= 223459

Congreso de la Nación Argentina. (2013). Ley $N^{\circ}$ 26.899. Recuperado de http://servicios.infoleg.gob.ar/infolegInte rnet/verNorma.do?id=223459 
CONICET Consejo Nacional de Investigaciones Científicas y Técnicas. (2015). Resolución del Directorio $N^{\circ} 2705$. Recuperado de https://ri.conicet.gov.ar/themes/CONICETDigital/RD\%2020150710-2705.pdf

CONICET. (2016). Resolución del Directorio $N^{\circ} 515$. Recuperado de https://www.conicet.gov.ar/wp-content/uplo ads/RD-20160307-0515.pdf

CONICET. (2017a). CONICET en cifras. Indicadores Destacados. Buenos Aires: CONICET. Recuperado de https ://cifras.conicet.gov.ar/publica/

CONICET. (2017b). Encuentro de Especialistas RI. Buenos Aires: CONICET.

CONICET. (2019). RI CONICET Digital. ¿Cómo es el ciclo de publicación? Recuperado de https://ri.conicet.gov.a $\mathrm{r} /$ page/ciclo

COAR Confederation of Open Access Repositories. (2020, 29 de enero). Communiqué: meeting participants agree to work together on a technical architecture for distributed peer review on repository resources. Recuperado de https://www.coar-repositories.org/news-updates/communique-meeting-participants-agree-to-work-togeth er-on-a-technical-architecture-for-distributed-peer-review-on-repository-resources/

CSIC Consejo Superior de Investigaciones Científicas (2019). Transparent ranking: institutional repositories by Google Scholar (May 2019). Recuperado de http://repositories.webometrics.info/en/node/32

Entrevista OCA. Secretaría General. Universidad Nacional de Córdoba. 29 de abril 2019.

Entrevista Oficina Técnica. Gerencia de Organización y Sistemas, Consejo Nacional de Investigaciones Científicas y Técnicas. 12 de abril 2019.

Entrevista Red Federal de Especialistas en el RI. Consejo Nacional de Investigaciones Científicas y Técnicas. 11 de julio 2019.

IAPCByA Instituto Académico Pedagógico de Ciencias Básicas y Aplicadas. Universidad Nacional de Villa María. (2014). Resolución $N^{\circ} 55$.

IAPCH Instituto Académico Pedagógico de Ciencias Humanas. Universidad Nacional de Villa María. (2014). Resolución $N^{\circ} 121$.

IAPCS Instituto Académico Pedagógico de Ciencias Sociales. Universidad Nacional de Villa María. (2014). Resolución $N^{\circ} 202$.

MINCYT Ministerio de Ciencia, Tecnología e Innovación Productiva. (2011). Resolución $N^{\circ} 469$. Recuperado de h ttp://www.biblioteca.mincyt.gob.ar/docs/res_be_469-11.pdf

MINCYT. (2016). Resolución $N^{\circ}$ 753-E. Recuperado de http://servicios.infoleg.gob.ar/infolegInternet/anexos/265 000-269999/267833/norma.htm

SECTIP Secretaría de Ciencia, Tecnología e Innovación Productiva. Ministerio de Educación, Ciencia y Tecnología. (2002). Resolución $N^{\circ}$ 253. Recuperado de http://www.biblioteca.mincyt.gob.ar/docs/Resolucion\%20253-02. pdf

Repositorio Institucional CONICET Digital. (2020). Estadísticas. Recuperado de https://ri.conicet.gov.ar/wp/esta disticas

SNRD Sistema Nacional de Repositorios Digitales. (2019). Directrices 2019. Recuperado de http://repositoriosdigit ales.mincyt.gob.ar/vufind/Content/directrices

SNRD. (2020, 20 de marzo). Búsqueda Avanzada. Recuperado de http://repositoriosdigitales.mincyt.gob.ar/vufind/

UNC Universidad Nacional de Córdoba. (2014). Resolución Rectoral $N^{\circ} 1714$. Recuperado de http://www.digesto.u nc.edu.ar/rectorado/rectorado/resolucion/1714_2014_1/?searchterm $=1714$.

UNC. (2016). Resolución Rectoral $N^{\circ}$ 1914. Recuperado de http://www.digesto.unc.edu.ar/rectorado/rectorado/res olucion/1914_2016_1/?searchterm $=1914$

UNC. (2017a). Resolución del Honorable Consejo Superior $N^{\circ}$ 1125. Recuperado de http://www.digesto.unc.edu.ar/c onsejo-superior/honorable-consejo-superior/resolucion/1125_2017/?searchterm=1125/2017

UNC. (2017b). Resolución del Honorable Consejo Superior $N^{\circ}$ 1365. Recuperado de http://www.digesto.unc.edu.ar/c onsejo-superior/honorable-consejo-superior/resolucion/1365_2017/?searchterm=1365

UNC. (2019a). Comunicación personal, 16 de abril. 
UNC. (2019b). Resolución del Honorable Consejo Superior $N^{\circ}$ 1938E. Recuperado de http://www.digesto.unc.edu.ar /consejo-superior/honorable-consejo-superior/resolucion/1938_2019/?searchterm=1938/2019

UNC. (2019c). Resolución Rectoral $N^{\circ} 2215 E$. Recuperado de http://www.digesto.unc.edu.ar/rectorado/rectorado/ resolucion/2215_2019/?searchterm $=2215$

\section{Notas}

1 La COAR (Confederation of Open Access Repositories) está analizando el potencial de una arquitectura común distribuida que conectaría la revisión por pares con los recursos en los repositorios (COAR, 2020).

2 Para más información sobre la experiencia piloto, véase la ponencia titulada Plan de Gestión de Datos en CONICET: análisis, experiencia y desafíos (Actis y Carlino, 2018). 